\title{
ANTICIPATORY POSTURAL ADJUSTMENTS IN CHILDREN WITH HEMIPLEGIA AND DIPLEGIA
}

\author{
Girolami, Gay L. ${ }^{1}$, Shiratori, Takako ${ }^{2}$, Aruin, Alexander S. ${ }^{3}$ \\ ${ }^{1}$ Department of Kinesiology and Nutrition, University of Illinois at Chicago, IL 60612, \\ ${ }^{2}$ Pathways Center, Glenview, IL 60026 \\ ${ }^{3}$ Department of Physical Therapy, University of Illinois at Chicago, IL 60612 and
}

Corresponding author:

Alexander S. Aruin, Ph.D.

Department of Physical Therapy (MC 898)

University of Illinois at Chicago

1919 W. Taylor St. (4 $4^{\text {th }}$ floor)

Chicago, IL 60612

Tel: (312) 355-0904

Fax: (312) 996-4583

Email: aaruin@uic.edu 


\section{ABSTRACT}

Anticipatory postural adjustments (APAs) play an important role in the performance of many activities requiring the maintenance of standing posture. However, little is known about how or if children with cerebral palsy (CP) generate APAs. Two groups of children with CP (hemiplegia and diplegia) and a group of children with typical motor development performed arm flexion and extension movements while standing on a force platform. Electromyographic activity of six trunk and leg muscles and displacement of center of pressure (COP) were recorded. Children with $\mathrm{CP}$ were able to generate anticipatory postural adjustments, produce directionally specific APAs and COP displacements similar to those described in adults and typically developing children. However, children with diplegia were unable to generate APAs of the same magnitude as children with typical development and hemiplegia and had higher baseline muscle activity prior to movement. In children with diplegia, $\mathrm{COP}$ was posteriorly displaced and peak acceleration was smaller during bilateral extension compared to children with hemiplegia. The outcomes of the study highlight the role of APAs in control of posture of children with CP and point out the similarities and differences in anticipatory control in children with diplegia and hemiplegia. These differences may foster ideas for treatment strategies to enhance APAs in children with CP. 


\section{INTRODUCTION}

Cerebral palsy (CP), a neurodevelopmental condition caused by a non-progressive brain lesion, can occur before, during or shortly after birth (Bax et al. 2005; Rosenbaum 2007).

Children diagnosed with $\mathrm{CP}$ demonstrate increased muscle activity to sustain posture, agonist/antagonist co-contraction, impaired postural control, inadequate force production, and restricted voluntary and selective control of movement (Campbell 1991; Damiano et al. 2001; Olney and Wright 2006; Prosser et al. 2010). These impairments not only interfere with performance of functional activities, but also with opportunities and/or willingness to participate in leisure, community and school activities (Roncesvalles et al. 2002; Olney and Wright 2006). Impaired postural control in $\mathrm{CP}$ includes difficulty organizing compensatory postural adjustments (CPAs) and anticipatory postural adjustments (APAs). CPAs make use of sensory feedback to respond to and counter disturbances of posture and balance and were first described with respect to children with typical development and children with CP in groundbreaking study by Nashner et al, (Nashner et al. 1983). This study provided the stimulus for research describing the development of CPAs in infants and children (Brogren et al. 1996; Hadders-Algra et al. 1998; Woollacott et al. 1998; van der Heide et al. 2004).

APAs are generated by the central nervous system (CNS) to counteract the expected mechanical effects of perturbation in a feedforward manner; they are described as the activation of in the trunk and legs at least $100 \mathrm{~ms}$ prior to a forthcoming predictable perturbation. Studies in adults have shown that APAs are associated with direction specific patterns of postural muscle activation or inhibition, as well as anticipatory center of pressure changes (Bouisset and Zattara 1981; Riach et al. 1987; Aruin and Latash 1995; Santos and Aruin 2008). At present, the 
equivalent breadth of descriptive research describing APAs in typically developing children and children with CP is unavailable.

The majority of APA studies involving children are limited to reports on anticipatory changes in center of pressure position prior to forward or backward arm reaching tasks performed in standing (Riach and Hayes 1990; Hay and Redon 1999; Hay and Redon 2001; Liu et al. 2007). These studies have shown that children can produce posterior and lateral COP displacements before and during reaching tasks but the younger children had delayed reaction time and greater variability of anticipatory anterior/posterior COP displacements than older children and adults.

Recently, it was reported that children with and without disabilities were able to produce anticipatory EMG and COP changes during bilateral shoulder flexion (Girolami et al. 2010; Tomita et al. 2010) and bilateral shoulder extension (Girolami et al. 2010) tasks performed in standing. However, no research to date has been designed to look at the diagnosis-related differences in APAs in children with CP.

The aim of this study was to investigate anticipatory EMG activity and COP changes in children with CP (hemiplegia and diplegia) associated with the performance of bilateral shoulder flexion (FLEX) and extension (EXT) performed in standing and to compare their performance to children with typical motor development. FLEX and EXT were selected because they represent functional upper extremity movements used by children during daily life activities (i.e. reaching forward or backward for toys/objects, throwing a ball etc.). Additionally, these movements are well represented in the adult APA literature (Zattara and Bouisset 1988; Aruin and Latash 1995) and form a basis for comparison. 
We hypothesized that children with $\mathrm{CP}$ will be able to produce direction specific APAs and changes in center of pressure (COP). We also hypothesized that there would be differences in baseline muscle activity and APAs between the children with typical development and the children with CP.

\section{METHODS}

\section{PARTICIPANTS}

Three groups of children between the ages of 7 and 17 were enrolled in this study. The first two groups were children with a diagnosis of cerebral palsy; spastic hemiplegia (HEMI, $n=9$ ) and spastic diplegia (DIPL, $\mathrm{n}=9$ ). The third group was children who were typically developing (TD) $(\mathrm{n}=9)$ (Table 1). To be eligible for the study, the children with CP met the following criteria: a medical diagnosis of CP, Gross Motor Function Classification of I or II (children who can stand and ambulate without the use of an assistive device), no surgical or Botox interventions for 6 months prior to enrollment and the ability to understand procedures and follow directions. The children with typical developing had no known motor, sensory visual or neurological conditions (see Table 1).

All participants were recruited following procedures approved by the University of Illinois at Chicago Institutional Review Board. Parental consent was obtained and each child was required to give both written and verbal assent to participate. The children with $\mathrm{CP}$ were recruited by contacting therapists and physicians in private practice, local hospitals, rehabilitation facilities, and out-patient clinics in the Chicago metropolitan area. The children with typical motor development were a sample of convenience recruited from among family, friends and coworkers.

$<$ Table 1 is about here $>$ 


\section{INSTRUMENTATION}

The subjects performed bilateral arm movement tasks while standing on a force platform (OR-6, AMTI, Inc., USA) that was used to record ground reaction forces and moments of force. A miniature unidirectional accelerometer (PCB Piezotronics, Inc, USA), with the axis of sensitivity oriented in the plane parallel to the direction of the arm movement was taped to the dorsal surface of the dominant hand and used to record the arm acceleration during the performance of the tasks.

Using locations identified from the literature (Basmajian 1980), surface EMG activity was recorded on the right and left side of the body by placing pairs of disposable pediatric electrodes (3M Company, MN) over the bellies of the following muscles: Rectus Abdominus (RA), Erector Spinae (ES), Rectus Femoris (RF), Biceps Femoris (BF), Tibalis Anterior (TA), and Medial Soleus (SOL). EMG signals were collected using a differential amplifier (gain 2000, $10-500 \mathrm{~Hz}$, analog filter) (RUN Technologies, USA). The EMG, force platform signals and accelerometer data were digitized using the analog-to-digital board with a 16 bit resolution at $1000 \mathrm{~Hz}$ and customized LabView software (National Instruments, TX) was used to collect the data and perform the offline data analysis.

\section{PROCEDURE}

Two experimental tasks, bilateral shoulder flexion and bilateral shoulder extension were studied (Fig. 1). The children were instructed to stand quietly on the force platform with feet placed shoulder width apart and a chalk outline was made around each foot to ensure consistent positioning during data collection. To stabilize the hand position and direct attention to the movement, the subjects held lightweight plastic tubes in each hand (42 $\mathrm{g}$ each). 
$<$ Figure 1 is about here $>$

Bilateral Shoulder Flexion (FLEX) was defined as forward arm movement executed to 90 degrees of shoulder flexion, while Bilateral Shoulder Extension (EXT) was competed to approximately 45 degrees of shoulder extension (See Fig 1). For all experimental trials, the participants were instructed to look directly forward and to perform each task "as fast as possible" in a self-paced mode after a computerized go-tone. They were instructed to execute 'sharp, clear movements', sustaining the ending position for $2-3$ seconds. For each experimental task, the participants performed 8 trials with a 5 second interval between each trial. The presentation order of the tasks was randomized across participants. The participants practiced each task $2-4$ times prior to the initiation of data collection.

To counter fatigue, participants could request a break to rest, drink or eat a snack any time during the data collection session. In addition, a two-minute imposed rest was scheduled between movements.

\section{DATA COLLECTION AND PROCESSING}

\section{EMG Data}

The EMG data were processed offline using customized LabView software. The process involved rectification and filtering of the EMG signals using a $100 \mathrm{~Hz}$ low pass, second order Butterworth filter and filtering of the platform and accelerometer signals using a second order Butterworth low pass filter set at $20 \mathrm{~Hz}$. Every trial for each of the two movements were viewed on a PC monitor and aligned by an experienced research based on the first deflection of the accelerometer trace. This deflection was labeled as the initiation of arm movement and identified as $\mathrm{T}_{0}$. The aligned trials for each movement were averaged for each subject. 
Baseline EMG integrals $\left(\int \mathrm{EMG}_{\mathbf{B L}}\right)$, defined as the period of EMG activity from $-500 \mathrm{~ms}$ to $-400 \mathrm{~ms}$ before $\mathrm{T}_{0}$, were obtained for two purposes. First they were necessary for the normalization of anticipatory integrals, but additionally we were interested in comparing baseline muscle activity among the three participant groups using the mean $\int E_{M G}$. Anticipatory EMG activity was quantified as the integral of EMG activity $\left(\int \mathrm{EMG}_{\mathbf{1 0 0}}\right)$ occurring from $-100 \mathrm{~ms}$ to time zero $\left(\mathrm{T}_{0}\right)$. The selection of this $100 \mathrm{~ms}$ period is consistent with the procedure used in other studies (Gantchev and Dimitrova, 1996, Shiratori and Latash, 2001).

To compare anticipatory EMG activity between the right and left muscles for all participant groups and between the two experimental tasks, it was necessary to normalize the resultant $\int E_{\text {EGG }} 100$. To allow comparisons between right and left sides for the same muscle group we calculated the mean $\int \mathrm{EMG}_{\mathrm{BL}}$ and mean $\int \mathrm{EMG}_{100}$ values by task for each muscle of each participant. These values were used to represent anticipatory muscle activity $\left(\int \mathrm{EMG}_{\mathrm{APA}}\right)$ with reference to a baseline activity as follows:

\section{$\left.\int E M G_{A F A}=\left(\operatorname{maan} E M G_{100}\right) \operatorname{mean} \int E M G_{B L}\right)^{\prime}$ Thus an $\int E M G_{A P A}$ value of 1 is the same} as baseline EMG value, while values above 1 denote an increase in baseline activity and values below 1 indicate a decrease or suppression of baseline activity.

\section{Peak Acceleration and COP Data}

Acceleration signals for each participant and for each task were averaged and value of first peak acceleration occurring between $\mathrm{T}_{0}$ and $+300 \mathrm{~ms}$ from $\mathrm{T}_{0}$ was obtained.

COP displacements in the anterior/posterior direction were calculated using the following

approximation: $C O P x=-\frac{M_{y}+F_{x} * d}{F_{z}}$ where $M_{Y}$ is the moment in sagittal plane, $F_{Z}$ and $F_{X}$ are the vertical and anterior-posterior components of the ground reaction force, and $d$ is the distance 
from the origin of the force platform to its surface (Winter et al. 1996). The COP $x$ data were averaged for each participant and for each FLEX and EXT task and anticipatory $\mathrm{COP}_{\mathrm{APA}}$ displacement was defined at time $T_{0}$ with regard to the respective baseline $(-500-400 \mathrm{~ms}$ before $\mathrm{T}_{0}$ ) from the above data.

\section{Statistical Analysis}

Mechanical characteristics, in particular speed of movement, have been shown to influence the APA magnitude (Horak et al. 1984; Woollacott and Manchester 1993). Peak acceleration was analyzed to assess consistency or inconsistency of shoulder movements between groups using one way Group (TD, HEMI, DIPL) Analysis of Variance (ANOVA) for each movement direction (FLEX, EXT). For further group comparison, independent $\mathrm{T}$ test was used.

Repeated measures ANOVA was used to compare $\mathrm{COP}_{\mathrm{APA}}$ data across groups and movement directions. To quantify whether $\mathrm{COP}_{\mathrm{APA}}$ changes were significantly different from baseline (zero) values for each condition and for each group, a one sample t-test was used.

EMGs $\left(\int \mathrm{EMG}_{\mathrm{APA}}, \int \mathrm{EMG}_{\mathrm{BL}}\right)$ were analyzed using multiple repeated measures ANOVAs with three main factors: Group, Body side (left, right), and Movement direction, with the $\int \mathrm{EMG}_{\mathrm{APA}}$ or $\mathrm{EMG}_{\mathrm{BL}}$ for each muscle as the dependent measures. However, statistical analysis showed no significant main effect or interaction effect with body side. Thus, data for right and left sides of the muscles were pooled and analyzed with repeated measures ANOVA for main effects of group and movement direction. For post hoc analyses, independent $\mathrm{T}$ tests were used to compare between groups, and dependent $\mathrm{T}$ tests were used to compare within group comparisons. Statistical significance was set at $\mathrm{p}<0.05$ and only those results significant at $\mathrm{p}<0.05$ and trends at $\mathrm{p}<0.1$ are reported. 


\section{RESULTS}

All participants were able to perform the two movements (FLEX and EXT).

\section{Peak Acceleration}

Peak acceleration values for three groups of children are indicated on Table 2. There was no significant difference in peak acceleration among the three groups of children for FLEX.

For EXT, between group comparisons using one way ANOVA showed a significant Group effect $\left(\mathrm{F}_{(2,24)}=3.71, \mathrm{p}<0.05\right)$ with significant difference between the TD and DIPL pair $(p=0.05)$.

$$
<\text { Table } 2 \text { is about here }>
$$

\section{EMG Activity}

EMG traces of the right and left trunk and upper leg muscles for a representative child from each group are presented in Figures 2 and 3. Anticipatory activation of muscles during FLEX (Fig 2) can be seen in the right and left ES and BF muscles in all three children. Bursts of activity in these muscles prior to $\mathrm{T}_{0}$ are clear for TD and HEMI children but more diffuse for the DIPL child.

During EXT, anticipatory muscle activity was observed in the right and left RA and RF in the TD child, accompanied by anticipatory inhibition in the right and left ES and BF muscles (Fig 3, left panels). In the children with $\mathrm{CP}$, anticipatory activity was observed in the RA muscles bilaterally, but the RF muscles showed smaller anticipatory activation in child with hemiplegia (Fig 3, middle panels) and no anticipatory EMG activity in the child with diplegia 
(Fig 3, right panels). Anticipatory inhibition of the ES and BF muscles is less prominent in the children with $\mathrm{CP}$.

Directional specificity of anticipatory muscle activity can be seen in all three groups as the activation of dorsal muscles during FLEX and activation of ventral muscles and inhibition of dorsal muscles during EXT.

$<$ Figures 2 and 3 are about here $>$

Anticipatory EMG integrals ( $\left(\mathrm{EMG}_{\mathrm{APA}}\right)$ for $\mathrm{ES}, \mathrm{BF}, \mathrm{RA}$ and RF muscles pooled from both right and left body sides are plotted for each group and each movement direction (FLEX and EXT) in Figure 4.

The results indicate a main effect of Movement Direction in $\mathrm{RA}\left(\mathrm{F}_{(1,51)}=43.29, \mathrm{p}<.0001\right)$, $\operatorname{ES}\left(\mathrm{F}_{(1,51)}=39.59, \mathrm{p}<.0001\right), \operatorname{BF}\left(\mathrm{F}_{(1,51)}=21.90, \mathrm{p}<.0001\right)$, and $\mathrm{RF}\left(\mathrm{F}_{(1,51)}=4.67, \mathrm{p}<.05\right)$.

Furthermore, there was a significant interaction between Group and Movement Direction in RA, $\left(\mathrm{F}_{(2,51)}=4.97, \mathrm{p}<.01\right), \mathrm{ES},\left(\mathrm{F}_{(2,51)}=4.85, \mathrm{p}<.01\right)$ and $\mathrm{BF}\left(\mathrm{F}_{(2,51)}=6.71, \mathrm{p}<.005\right)$

During EXT, the TD group $(\mathrm{p}<.01)$ exhibited greater APA activity in the ES muscles than the HEMI and DIPL groups. During EXT, TD $(p<.01)$ and HEMI $(p<.01)$ groups were able to produce larger APA activity in RA than DIPL and suppression of baseline activity in BF was greater in TD compared to the groups with HEMI $(\mathrm{p}<.005)$ and DIPL $(\mathrm{p}<.001)$.

$<$ Figure 4 is about here $>$

\section{Baseline Activity}

Visual inspection of individual muscle traces revealed baseline EMG activity was higher for some of the children with CP than for children in the TD group (see Figs 2 and 3). Therefore, 


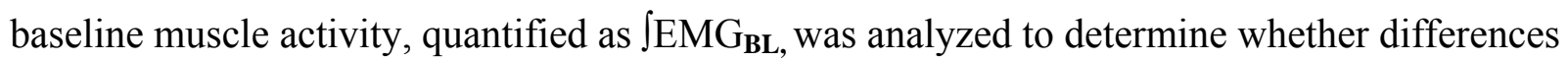
in the magnitude of the baseline activity for group, body side, and movement direction were significant. There was no main effect or interaction for body side in any of the muscle groups. Thus the $\mathrm{EMGG}_{\mathrm{BL}}$ for right and left muscles were pooled for each muscle and repeated measures ANOVA was rerun for Group and Movement Direction.

There was a significant difference in baseline values between groups (main effect of Group: $\operatorname{ES}\left(\mathrm{F}_{(1,51)}=3.23, \mathrm{p}<.05\right), \operatorname{RF}\left(\mathrm{F}_{(1,51)}=21.79, \mathrm{p}<.0001\right)$, and $\mathrm{BF}\left(\mathrm{F}_{(1,51)}=4.12, \mathrm{p}<.05\right)$. In particular baseline values were higher for the DIPL group for $\mathrm{ES}(\mathrm{p}<.05), \mathrm{RF}(\mathrm{P}<.0001), \mathrm{BF}$ $(\mathrm{p}<.05)$ as compared to TD group and for $\mathrm{RF}(\mathrm{p}<.0001)$ and $\mathrm{BF}(\mathrm{p}<.01)$ as compared to the Hemi group. However, the baseline activity of the RA muscles $\left(\mathrm{F}_{(1,51)}=11.18, \mathrm{p}<.0001\right)$ was significantly higher in the TD group than in the HEMI $(\mathrm{p}<.0001)$ or DIPL $(\mathrm{p}<.0002)$ groups.

$<$ Figure 5 is about here $>$

\section{Center of Pressure Displacement}

Anticipatory changes in COP specific to movement direction were observed in the TD and HEMI groups, but not in the DIPL group (interaction effect of GROUP and MOVEMENT DIRECTION, $\left.\mathrm{F}_{(2,24)}=3.86, \mathrm{p}<.05\right)$. For all of the groups, the posterior shift in the COP was observed prior to FLEX, in the direction opposite the arm movement. However, post hoc one way t-tests revealed a significant difference of the COP from the baseline phase (-500 to $-400 \mathrm{~ms}$ prior to movement initiation) for the TD group $\left(\mathrm{t}_{(8)}=-4.259, \mathrm{p}<.05\right)$, but not for the HEMI and DIPL groups.

Prior to EXT, the COP displacement was anterior in the TD and HEMI groups, but posterior in the DIPL group (Fig 6). Post-hoc one way t-test revealed that the anterior COP 
change prior to the initiation of EXT was significantly different from baseline phase in the TD group $\left(\mathrm{t}_{(8)}=8.99, \mathrm{P}<0.0001\right)$, close to significant for HEMI group $\left(\mathrm{t}_{(8)}=2.14, \mathrm{p}=0.065\right)$, but not significant for DIPL. It is important to note that for both movements, the TD group showed the largest anticipatory COP displacements.

$<$ Figure 6 is about here $>$

\section{DISCUSSION}

The main findings of our study indicate that children with typical motor development and children with $\mathrm{CP}$, as young as seven years of age, were able to produce directionally-specific anticipatory muscle activity during FLEX and EXT movements. However, the responses in the children with $\mathrm{CP}$ were more variable than TD performance showing less consistent anticipatory muscle activity in the upper legs for both FLEX and EXT. Further, direction specific anticipatory COP displacement was present in TD and HEMI but not in DIPL. Finally, the DIPL group had increased baseline EMG activity, which may have compromised their ability to generate anticipatory EMG activity at the same levels as the TD and HEMI groups.

\section{Peak Acceleration}

Children with cerebral palsy have poor postural and selective motor control as a result of altered muscle tone and deficits in neuromuscular activation (Prosser et al. 2010). The significantly slower peak acceleration in the DIPL group during EXT may be explained by the standing alignment adopted by the children. Another explanation might be poor selective control of the upper extremity and/or decreased ability to practice shoulder extension movements. 
With respect to alignment, two postures were observed in the DIPL group during quiet standing; crouching $(n=4)$ and lumbar lordosis $(n=5)$. These postures, frequently described in children with diplegia (Gage 1993; Damiano et al. 1995; Hicks et al. 2008) and are thought to improve postural stability by decreasing degrees of freedom in the trunk and lower extremities (Damiano et al. 2006; Prosser et al. 2010). However, it has been speculated that reducing degrees of freedom may improve stability but may simultaneously constrain the ability to make fine adjustments in the trunk and extremities in response to the environment or external perturbations (Prosser et al. 2010). If this is the case, the CNS may have acted to reduce upper extremity acceleration to minimize the threat to stability on standing. The strategy of reducing arm speed has been reported in adults under conditions of postural instability (Shiratori and Latash 2000). Therefore, we recommend that future APA studies explore the effects of alignment on upper extremity acceleration. In addition, care should be taken to train APAs in children with $\mathrm{CP}$ under conditions of optimal postural alignment. This may include using video tapes to demonstrate correct posture, verbal cues and interventions to address muscle strengthening to achieve more optimal trunk and lower limb alignment.

With respect to selective motor control and practice, reaching studies in children with $\mathrm{CP}$ have shown that the trajectory of the arm is irregular and accompanied by decreased speed and smoothness of movement as compared to children without CP (Eliasson et al. 1991; Eliasson et al. 2006). However, improvements in these parameters have been reported following training (Kluzik et al. 1990; Eliasson et al. 1991; Harbourne 2001).

These finding support the need for upper extremity practice to improve speed and fluidity of movement. We recommend that treatments to improve APAs in children with $\mathrm{CP}$, and specifically children with diplegia, incorporate opportunities to practice bilateral and unilateral 
shoulder movements under stable postural conditions (e.g. sitting) prior to practicing these movements as part of the self-induced perturbations.

\section{APAs during Bilateral Shoulder Flexion and Bilateral Shoulder Extension}

In this study, children in the TD group and children with $\mathrm{CP}$ were generally able to generate directionally-specific APAs similar to those reported in healthy adults and children with typical motor development under like conditions (Aruin and Latash 1995; Girolami et al. 2010). During FLEX, the dorsal muscle groups of the trunk (ES) and upper leg (BF) were active prior to the perturbation restricting forward movement of the body and preserving balance. During EXT, anticipatory muscle activity was observed in the ventral muscle groups of the trunk (RA) and upper legs (RF) restricting backward movement of the body. The results demonstrate that despite CNS injury, children with CP (HEMI and DIPL) who ambulate without assistive devices, were able to organize directionally-specific anticipatory postural adjustments in muscles of the trunk and upper legs prior FLEX and EXT movements performed in standing.

Although able to generate directionally specific APAs, during both FLEX and EXT, the APAs of children with diplegia were smaller in magnitude than APAs in the TD and HEMI groups (see Fig 3 and 4) particularly in the RA muscles. Tomita et al (Tomita et al. 2010) reported a similar finding in a study of TD and DIPL children.

It has been shown that APA magnitudes scale with the magnitude of the perturbation (Aruin and Latash 1996) and slower arm movements produce smaller perturbations (Horak et al. 1984; Lee et al. 1987). We found the magnitude of the APAs was smaller in the RA muscles of the DIPL group during EXT. This may be related, in part, to the decreased acceleration of the upper extremity during EXT, along with the reduced fine tuning of muscle activity secondary to crouching and lordosis. It was reported that children with typical development, who assumed a 
crouch position during platform perturbations, had altered compensatory EMG activity which closely resembled the EMGs of children with diplegia (Burtner et al. 1998). We recommend that future studies investigate the effects of alignment and upper extremity acceleration on the magnitude of APAs and treatments to improve anticipatory posture in children with CP incorporate strategies to address alignment and selective upper extremity control.

\section{Baseline EMG Activity}

Excessive muscle activity, even at rest, has been reported in children with CP (Unnithan et al. 1996; Policy et al. 2001; Prosser et al. 2010) and is thought to be used as a means of improving postural control and stability (Nicholson et al. 2001; Rosenbaum et al. 2007). Although this study focused on APAs in children with CP, we also felt it would be important to analyze the baseline muscle activity to determine whether diminished postural stability might be reflected by differences in muscle activity during quiet standing. Baseline muscle activity has not been previously described in studies of APAs in adults or children.

We found that baseline EMG activity measured from -500-to -400ms prior to FLEX and EXT movements were higher in children with DIPL than in the TD and HEMI children for the ES, BF and RF muscles. One explanation might be an influence of the crouching and lordosis postures observed in the DIPL group (see peak acceleration and COP sections). Perhaps these postures, which minimize degrees of freedom were employed to enhance postural stability, but at a subsequent cost of increased baseline activity. Further, it is possible that the increased baseline muscle activity depleted the available motor neurons pool, which resulted in APAs of lower magnitude than those generated by the TD and Hemi groups. Measuring joint angles at the hip and knee would clarify the role of increased baseline activity with respect to alignment 
(crouching and lordsis) in individuals with CP. This speaks to the need for a future study incorporating both the body kinematics and EMG recordings.

The results of the current study indicated that children diagnosed with different types CP showed differences in their APAs and in their baseline muscle activity. In this case, the children with higher baseline muscle activity also showed a decrease in the magnitude of the anticipatory EMGs. We feel this finding lends additional credence to the previous recommendation (Damiano et al. 2006) that children with hemiplegia and diplegia, even those within the same GMFCS level, not be considered a homogeneous group when recruited for research studies

\section{Directional Specificity of Anticipatory COP Displacement}

For FLEX, the change in COP was consistent with the posterior displacements reported in adults and children during similar bilateral shoulder flexion tasks (Riach and Hayes 1990; Liu et al. 2007; Girolami et al. 2010; Tomita et al. 2010). For EXT, the TD and HEMI groups showed anterior COP displacements as previously reported in typical children and adults (Riach and Hayes 1990; Aruin and Latash 1995; Liu et al. 2007; Girolami et al. 2010). However, the mean COP displacement in the DIPL group was posterior.

More importantly than direction, is perhaps the amount of COP displacement shown by DIPL group, which was significantly smaller than displacements generated by the TD and HEMI groups. Similar reductions in anterior-posterior COP displacements were reported by Hsue and colleagues (Hsue et al. 2009) in a study of gait in children with CP. They speculated that excessively activating muscles of the trunk and hips might be a strategy employed to maintain upright posture against gravity. However, this excessive muscle activation, a compensation for poor postural control, limits the precise control of muscle activity for dynamic postural responses. 
This same assumption may be applied to our hypothesis regarding the possible effects of crouching and lordosis. These postures cause increased activity on the trunk and lower limb muscles, which may result in decreased ability to precisely control the responses to perturbations. Therefore, the CNS may respond by limiting the excursion of the COP to prevent a loss of balance.

These results, the first to document that children with diplegia displace the COP posteriorly during bilateral shoulder extension, should be further studied to delineate the effect of atypical alignment from the effect of the small sample size and variable responses of the participants. Also additional evidence is needed for development of a treatment protocol to improve postural alignment prior to training APAs in children with CP.

\section{CONCLUSION}

The results of the study indicate that by at least age seven, children with hemiplegia and diplegia are able to generate directionally specific anticipatory postural adjustments when performing bilateral shoulder flexion or bilateral shoulder extension in standing. However, magnitudes of APAs were smaller for groups with CP compared to the TD children.

Participants in each of the three groups showed a posterior COP displacement during FLEX. Responses for EXT were not consistent across all groups: TD and HEMI generated anterior COP, while the children with diplegia showed posterior COP displacement. Such a discrepancy might be attributed to differences in standing alignment among the three groups of children and should be further investigated with the focus of developing APA-based training protocols under conditions of optimal postural alignment. 
In addition, analysis of baseline muscle activity in the three participant groups revealed that the children with diplegia had higher baseline muscle activity during quiet stance and lower amplitude APAs.

\section{ACKNOWLEDGEMENTS}

We thank the children and families who participated in this study. We also thank Daisy Tan, PT for testing and assigning GMFCS levels to the participants with CP. We are grateful to S. Joshi, PT, MS, X. Li, PhD, M. Santos, PT, PhD and V. Iyengar, PT, MS for assistance with data collection. This study was supported in part by a grant from the Neuro-Developmental Treatment Association, Laguna Beach, CA.

\section{REFERENCES}

Aruin AS, Latash ML (1995) Directional specificity of postural muscles in feed-forward postural reactions during fast voluntary arm movements. Exp Brain Res 103: 323-332

Aruin AS, Latash ML (1996) Anticipatory postural adjustments during self-initiated perturbations of different magnitude triggered by a standard motor action. Electroencephalogr Clin Neurophysiol 101: 497-503

Basmajian JV (1980) Electromyography--dynamic gross anatomy: a review. Am J Anat 159: $245-260$ 
Bax M, Goldstein M, Rosenbaum P, Leviton A, Paneth N, Dan B, Jacobsson B, Damiano D (2005) Proposed definition and classification of cerebral palsy, April 2005. Dev Med Child Neurol 47: 571-576

Bouisset M, Zattara M (1981) A sequence of postural reactions precedes voluntary movements. Neurosci Lett 22: $263-270$

Brogren E, Hadders-Algra M, Forssberg H (1996) Postural control in children with spastic diplegia: muscle activity during perturbations in sitting. Dev Med Child Neurol 38: 379388

Burtner PA, Qualls C, Woollacott MH (1998) Muscle activation characteristics of stance balance control in children with spastic cerebral palsy. Gait Posture 8: 163-174

Campbell SK (ed) (1991) Pediatric Neurologic Physical Therapy. Churchill Livingston Publishing Co

Damiano D, Abel M, Romness M, Oeffinger D, Tylkowski C, Gorton G, Bagley A, Nicholson D, Barnes D, Calmes J, Kryscio R, Rogers S (2006) Comparing functional profiles of children with hemiplegic and diplegic cerebral palsy in GMFCS Levels I and II: Are separate classifications needed? Dev Med Child Neurol 48: 797-803

Damiano DL, Kelly LE, Vaughn CL (1995) Effects of quadriceps femoris muscle strengthening on crouch gait in children with spastic diplegia. Phys Ther 75: 658-667; discussion 668671

Damiano DL, Martellotta TL, Quinlivan JM, Abel MF (2001) Deficits in eccentric versus concentric torque in children with spastic cerebral palsy. Med Sci Sports Exerc 33: 117122 
Eliasson AC, Forssberg H, Hung YC, Gordon AM (2006) Development of hand function and precision grip control in individuals with cerebral palsy: a 13-year follow-up study. Pediatrics 118: e1226-1236

Eliasson AC, Gordon AM, Forssberg H (1991) Basic co-ordination of manipulative forces of children with cerebral palsy. Dev Med Child Neurol 33: 661-670

Gage JR (1993) Gait analysis. An essential tool in the treatment of cerebral palsy. Clin Orthop Relat Res: 126-134

Girolami GL, Shiratori T, Aruin AS (2010) Anticipatory postural adjustments in children with typical motor development. Exp Brain Res 205

Hadders-Algra M, Brogren E, Forssberg H (1998) Development of postural control--differences between ventral and dorsal muscles? Neurosci Biobehav Rev 22: 501-506

Harbourne RT (2001) Accuracy of movement speed and error detection skills in adolescents with cerebral palsy. Percept Mot Skills 93: 419-431

Hay L, Redon C (1999) Feedforward versus feedback control in children and adults subjected to a postural disturbance. Exp Brain Res 125: 153-162

Hay L, Redon C (2001) Development of postural adaptation to arm raising. Exp Brain Res 139: $224-232$

Hicks JL, Schwartz MH, Arnold AS, Delp SL (2008) Crouched postures reduce the capacity of muscles to extend the hip and knee during the single-limb stance phase of gait. J Biomech 41: 960-967

Horak FB, Esselman P, Anderson ME, Lynch MK (1984) The effects of movement velocity, mass displaced, and task certainty on associated postural adjustments made by normal and hemiplegic individuals. J Neurol Neurosurg Psychiatry 47: 1020-1028 
Hsue BJ, Miller F, Su FC (2009) The dynamic balance of the children with cerebral palsy and typical developing during gait. Part I: Spatial relationship between COM and COP trajectories. Gait Posture 29: 465-470

Kluzik J, Fetters L, Coryell J (1990) Quantification of control: a preliminary study of effects of neurodevelopmental treatment on reaching in children with spastic cerebral palsy. Phys Ther 70: 65-76; discussion 76-68

Lee WA, Buchanan TS, Rogers MW (1987) Effects of arm acceleration and behavioral conditions on the organization of postural adjustments during arm flexion. Exp Brain Res 66: $257-270$

Liu W, Zaino CA, Westcott McCoy S (2007) Anticipatory Postural Adjustments in Children with Cerebral Palsy and Children with Typical Development. (Pediatr Phys Ther 19:: $188-195$

Nashner LM, Shumway-Cook A, Marin O (1983) Stance posture control in select groups of children with cerebral palsy: deficits in sensory organization and muscular coordination. Exp Brain Res 49: 393-409

Nicholson JH, Morton RE, Attfield S, Rennie D (2001) Assessment of upper-limb function and movement in children with cerebral palsy wearing lycra garments. Dev Med Child Neurol 43: 384-391

Olney S, Wright S (2006) Cerebral Palsy. In: Campbell SK (ed) Physical Therapy for Children. WB Saunders Co., Philadelphia, PA, pp 625-664

Policy JF, Torburn L, Rinsky LA, Rose J (2001) Electromyographic test to differentiate mild diplegic cerebral palsy and idiopathic toe-walking. J Pediatr Orthop 21: 784-789 
Prosser LA, Lee SC, VanSant AF, Barbe MF, Lauer RT (2010) Trunk and hip muscle activation patterns are different during walking in young children with and without cerebral palsy. Phys Ther 90: 986-997

Riach C, Lucy SD, Hayes KC (1987) Adjustments to posture prior to arm movement. In: Jonsson B (ed) International series on biomechanics, Biomechanic X-A. Human Kinetics, Champaign, IL, pp 459-463

Riach CL, Hayes KC (1990) Anticipatory postural control in children. J Mot Behav 22: 250-266 Roncesvalles MN, Woollacott MW, Burtner PA (2002) Neural factors underlying reduced postural adaptability in children with cerebral palsy. Neuroreport 13: 2407-2410

Rosenbaum P (2007) The natural history of gross motor development in children with cerebral palsy aged 1 to 15 years. Dev Med Child Neurol 49: 724

Rosenbaum P, Paneth N, Leviton A, Goldstein M, Bax M, Damiano D, Dan B, Jacobsson B (2007) A report: the definition and classification of cerebral palsy April 2006. Dev Med Child Neurol Suppl 109: 8-14

Santos MJ, Aruin AS (2008) Role of lateral muscles and body orientation in feedforward postural control. Exp Brain Res 184: 547-559

Shiratori T, Latash M (2000) The roles of proximal and distal muscles in anticipatory postural adjustments under asymmetrical perturbations and during standing on rollerskates. Clin Neurophysiol 111: 613-623

Tomita H, Fukaya Y, Honma S, Ueda T, Yamamoto Y, Shionoya K (2010) Anticipatory postural muscle activity associated with bilateral arm flexion while standing in individuals with spastic diplegic cerebral palsy: A pilot study. Neurosci Lett 479: 166-170 
Unnithan VB, Dowling JJ, Frost G, Volpe Ayub B, Bar-Or O (1996) Cocontraction and phasic activity during GAIT in children with cerebral palsy. Electromyogr Clin Neurophysiol 36: $487-494$

van der Heide JC, Begeer C, Fock JM, Otten B, Stremmelaar E, van Eykern LA, Hadders-Algra M (2004) Postural control during reaching in preterm children with cerebral palsy. Dev Med Child Neurol 46: 253-266

Winter DA, Prince F, Frank JS, Powell C, Zabjek KF (1996) Unified theory regarding A/P and M/L balance in quiet stance. J Neurophysiol 75: 2334-2343

Woollacott MH, Burtner P, Jensen J, Jasiewicz J, Roncesvalles N, Sveistrup H (1998) Development of postural responses during standing in healthy children and children with spastic diplegia. Neurosci Biobehav Rev 22: 583-589

Woollacott MH, Manchester DL (1993) Anticipatory postural adjustments in older adults: are changes in response characteristics due to changes in strategy? J Gerontol 48: M64-70

Zattara M, Bouisset S (1988) Posturo-kinetic organisation during the early phase of voluntary upper limb movement. 1. Normal subjects. J Neurol Neurosurg Psychiatry 51: 956-965 


\section{FIGURE CAPTIONS}

Fig. 1 Schematic illustrations of the two experimental movements: A. Bilateral Shoulder Flexion (FLEX: Right and Left arms move simultaneously into shoulder flexion); B. Bilateral Shoulder Extension (EXT: Right and Left arms move simultaneously into shoulder extension). Force platform and electrode and accelerometer placements are illustrated in A.

Fig. 2 Averaged EMG traces for FLEX for representative participants from of each of the groups: A. Typically Developing (TD), B. Hemiplegia (HEMI), C. Diplegia (DIPL). The vertical line $\left(\mathrm{T}_{0}\right)$ represents the onset of arm movement and the point of alignment. Both right and left muscle groups are plotted and EMG scales are in arbitrary units. Dorsal muscles (ES, BF) are shown inverted for ease of comparison and their scales are on the right. Muscle activity in the TA and SOL muscles is unremarkable and therefore not shown.

Fig. 3 Averaged EMG traces for EXT for representative participants from each of the three groups, TD, HEMI, and DIPL. For details see Fig. 2. Muscle activity in the TA and SOL muscles is unremarkable and therefore not shown.

Fig.4 Normalized Integrals of anticipatory changes in muscle activity were pooled for both the right and left body sides and averaged across the nine participants in each of the three groups for FLEX and EXT. Dorsal muscles are depicted in the left panels and ventral muscles in the right panels. Mean values and standard deviation bars are shown. In these plots, baseline activity is 
defined as 1; activation is a value $>1$; suppression is a value $<1$. Significant interaction effect of movement GROUP x movement direction is indicated with * $(\mathrm{p}<0.05)$.

Fig. 5 Normalized EMG integrals for baseline muscle activity averaged across the nine participants in each of the three groups for both FLEX and EXT. Dorsal muscle groups are on the left and ventral muscle groups on the right. Mean values and standard deviation bars are shown. Asterisks denote statistical significance between DIPL and * (TD) and ** (HEMI) groups. Plus sign denotes statistical significance between TD and ${ }^{+}$(HEMI) and ${ }^{++}$(DIPL) groups. Significance level is $\mathrm{p}<0.05$.

Fig. 6 Anticipatory changes in COP prior to FLEX and EXT, plotted for each participant group. Mean values and standard deviation are shown. Asterisks $(*)$ denote significant change in COP from the baseline $(\mathrm{p}<.05)$. 